\title{
Smarter treatment options to tackle the silent menace of Obesity and Obstructive Sleep Apnoea (OSA)
}

\author{
Journal of Diabetes and Endocrinology Research
}

Editorial Article

\section{Dr Mahender Yadagiri, Dr Parijat De*}

\author{
Department of Diabetes \& Endocrinology, City Hospital, \\ Birmingham UK
}

\author{
Correspondence author \\ Dr Parijat De \\ Department of Diabetes \& Endocrinology \\ City Hospital \\ Birmingham UK \\ Tel : :01215074104 \\ E-mail : p.de@nhs.net
}

Submitted : 20 Nov 2019 ; Published : Nov 2019
Obesity is a rapidly growing global public health issue in view of its prevalence, costs, and complications. As per WHO in 2016, the worldwide prevalence of obesity $\left(\mathrm{BMI}>30 \mathrm{Kg} / \mathrm{m}^{2}\right)$ is about $13 \%$ of the world's adult population (11\% of men and $15 \%$ of women). The worldwide prevalence of obesity nearly tripled between 1975 and 2016. 29\% of adults were classified as obese in England 2019, an increase from $26 \%$ in 2016 [1].

The prevalence of OSA in adults, is estimated to be $25 \%$ and as high as $45 \%$ in obese subjects [2]. If obesity is left untreated, there is an increased risk of type 2 diabetes, obstructive sleep apnoea, coronary heart disease, hypertension, stroke, cancer, osteoarthritis apart from psychological effects as well as causing huge economic burden to nations [3].

There is an urgent need for management options which are less invasive and more effective. Obesity imposes mechanical loads on both the upper airway and respiratory system that predispose to upper airway narrowing, collapse and airflow obstruction during sleep. Continuous positive airway pressure (CPAP) is the leading therapy for treating OSA but it's not comfortable to wear overnight.

Although bariatric surgery is effective in achieving the maximum weight loss and remission of conditions associated with obesity, there is always a risk of post-operative complications, prolonged inpatient admission and healthcare costs. Endoscopic bariatric therapy (EBT) is safe, cost effective and minimally invasive treatment option [4]. For those patients who don't fit BMI criteria for bariatric surgery or failed conservative or pharmacotherapy, EBT would be ideal.

Currently, approved EBT devices are gastric therapies like intra gastric balloons (Orbera, Reshape, Obalon), aspiration therapy (The Aspiration Assist system) along with suturing and plicating devices. There are multiple small bowel EBT's under investigation like duodenal-jejunal bypass liners (Endobarrier), duodenal mucosal resurfacing (DMR) and dual path enteral bypass [5]. Most of these treatment options are insitu for 6-12 months only, accelerating the benefits both in terms of weight and metabolic control.

Thereis anongoingresearch trial \{End-OSA(ISRCTN:33788132)\} involving Endobarrier to assess the extent to which patients with type 2 diabetes/pre-diabetes, obesity (BMI $30-45 \mathrm{Kg} / \mathrm{m}^{2}$ ) and moderate OSA requiring CPAP are able to discontinue CPAP following Endobarrier related weight loss. It is a response to intervention trial involving 10 subjects with a study duration of 24 months and at the recent data analysis (after 21 months follow up), all patients had apnoea hypopnea index (AHI) $<15$ events/ hour suggesting mild OSA and hence, they all have remained off CPAP [6].

As endoscopy units are ubiquitous all over the world, there should be a push towards offering smarter endoscopic bariatric therapies to tackle the twin global epidemic of obesity and obstructive sleep apnoea.

\section{References}

1. NHS Digital Statistics on Obesity (2019) Physical Activity and Diet, England 2019 http://digital.nhs.uk/pubs/sopad19

2. A Romero-Corral et al (2010) Interactions between obesity and obstructive sleep apnoea; Chest/137/3/March 2010; doi: 10.1378 chest. $09-0360$

3. Agha et al (2017) The rising prevalence of obesity; International Journal of Surgical Oncology 2: 17.

4. V Shahnazarian et al (2019) Endoscopic bariatric therapies for treating obesity; Translational Gastroenterology and Hepatology 4: 16.

5. S Sullivan (2017) Endoscopic Medical Devices for Primary Obesity Treatment in Patients with Diabetes; Diabetes Spectrum 30: 258-264.

6. Yadagiri et al (2019) Endobarrier in T2DM/pre-diabetes with obstructive sleep apnoea study ; Maintenance of improvement after Endobarrier removal; 2080-Poster presentation ADA 2019, San Francisco; Diabetes 68. 